\title{
Characteristics and management of patients with inflammatory bowel disease between a secondary and tertiary hospitals: a propensity score analysis
}

\author{
Ki Hwan Song ${ }^{1}$, Eun Soo Kim², Yoo Jin Lee ${ }^{3}$, Byung Ik Jang ${ }^{4}$, Kyeong Ok Kim ${ }^{4}$, Sang Gyu Kwak ${ }^{5}$, \\ Hyun Seok Lee ${ }^{2}$, on behalf of Crohn's and Colitis Association in Daegu-Gyeongbuk (CCAiD) \\ ${ }^{1}$ Department of Surgery, Goo Hospital, Daegu, ${ }^{2}$ Department of Internal Medicine, School of Medicine, Kyungpook National University, Daegu, \\ ${ }^{3}$ Department of Internal Medicine, Keimyung University School of Medicine, Daegu, ${ }^{4}$ Department of Internal Medicine, Yeungnam University \\ College of Medicine, Daegu, ${ }^{5}$ Department of Medical Statistics, Catholic University of Daegu School of Medicine, Daegu, Korea
}

Background/Aims: This study aimed to compare the clinical characteristics and management patterns of inflammatory bowel disease (IBD) patients in a secondary hospital (SH) with those in tertiary referral centers (TRC). Methods: Data from IBD patients in SH and 2 TRCs were retrospectively reviewed. The cumulative thiopurine use rate was compared between hospitals after controlling for different baseline characteristics using propensity score matching. Results: Among the total of 447 patients with IBD, 178 Crohn's disease (CD) and 269 ulcerative colitis (UC) patients were included. Regarding initial CD symptoms, patients from SH were more likely to show perianal symptoms, such as anal pain or discharge ( $56.6 \% \mathrm{vs.} 34.3 \%, P=0.003)$, whereas those from TRCs more often had luminal symptoms, such as abdominal pain ( $54.9 \%$ vs. $17.1 \%, P<0.001)$, diarrhea ( $44.1 \%$ vs. $18.4 \%, P<0.001)$, and body weight loss $(9.8 \%$ vs. $1.3 \%, P=0.025)$. Complicating behaviors, such as stricturing and penetrating, were significantly higher in TRCs, while perianal disease was more common in SH. Ileal location was more frequently observed in TRCs. For UC, SH had a more limited extent of disease (proctitis $58.8 \%$ vs. $21.2 \%, P<0.001$ ). The cumulative azathioprine use rate in SH was significantly lower than that in TRCs in both CD and UC patients after controlling for disease behavior, location, and perianal disease of CD and extent of UC. Conclusions: The clinical characteristics and management of the IBD patients in SH were substantially different from those in TRCs. Thiopurine treatment was less commonly used for SH patients. (Intest Res 2018;16:216-222)

Key Words: Inflammatory bowel disease; Secondary care; Tertiary care centers; Propensity score

\section{INTRODUCTION}

Inflammatory bowel disease (IBD), which mainly comprises UC and CD, is characterized by chronic repetitive bowel inflammation that requires life-long treatment. ${ }^{1}$ Although IBD prevails in Western countries, recent stud-

Received May 2, 2017. Revised August 13, 2017

Accepted August 14, 2017. Published online September 15, 2017

Correspondence to Eun Soo Kim, Division of Gastroenterology and Hepatology, Department of Internal Medicine, School of Medicine,

Kyungpook National University, 680 Gukchaebosang-ro, Jung-gu, Daegu

41944, Korea. Tel: +82-53-200-5879, Fax: +82-53-200-5879, E-mail:

dandy813@hanmail.net ies have shown that the incidence of the disease has significantly increased over the past several decades in Asia. ${ }^{2,3}$ This change in Asia may be the result of Westernized diet, improved hygiene, increasing use of antibiotics, or changes in the gut microbiota. ${ }^{4}$

IBD is a unique and disabling condition that often demands the care of gastroenterology specialists. Indeed, many studies on IBD in the literature have been conducted in tertiary referral centers (TRCs). ${ }^{5}$ However, little is known about the clinical features and management of patients with IBD in primary or secondary care units. We surmise that there must be a difference in the characteristics and treatments between patients in secondary hospitals (SHs) and those in

๑ Copyright 2018. Korean Association for the Study of Intestinal Diseases. All rights reserved.

This is an Open Access article distributed under the terms of the Creative Commons Attribution Non-Commercial License (http://creativecommons.org/licenses/by-nc/4.0)

which permits unrestricted non-commercial use, distribution, and reproduction in any medium, provided the original work is properly cited. 
TRCs. It would be clinically relevant to understand if there are any disparities for better understanding of IBD patients as a whole and for setting up an appropriate management strategy for patients who were referred from primary or SHs. Therefore, we aimed to compare the demographic and clinical characteristics of IBD patients from a SH with those from TRCs. The steroid, immunomodulator and anti-tumor necrosis factor (anti-TNF) use pattern for patients with IBD was also compared between the 2 groups.

\section{METHODS}

\section{Subjects}

The medical records of patients with CD and UC from an SH and 2 TRCs were reviewed retrospectively by 2 gastroenterology specialists (E.S.K. and K.O.K.). The SH had general physicians and surgeons while the 2 TRCs had specialized gastroenterology units including the department of gastroenterology and the department of surgery and only received referred patients from primary or SHs. Their diagnoses were made between January of 2011 and March of 2015. Patients older than 18 years and who were followed up with at least more than 6 months after diagnosis were included in the study. The disease diagnosis was based on a thorough clinical history taking, physical examination, laboratory investigations, endoscopic assessment, radiologic findings, and pathology assessment according to the Korean guidelines of IBD diagnosis. ${ }^{6,7}$

The Institutional Review Boards from all of the hospitals approved this study (DSMC2015-06-003).

The informed consent from patients was waived because this was a retrospective study.

\section{Variables}

Variables included age at diagnosis, sex, initial symptoms and history of medications, such as steroid exposure, thiopurine, and anti-TNF. Initial CD symptoms included perianal symptoms, such as anal discharge or painful anal swelling, and luminal symptoms, such as abdominal pain, diarrhea, or body weight loss. The UC symptoms consisted of hematochezia, diarrhea, abdominal pain, or body weight loss. For $\mathrm{CD}$, the locations were classified as ileal (L1), colonic (L2) or ileocolonic (L3), and the behaviors were categorized as inflammatory (B1), stricturing (B2) or penetrating (B3) in correspondence with the Montreal classification. Perianal disease, including perianal fistula or abscess, was also recorded.
However, hemorrhoids and skin tag were not included in the definition of perianal disease. For UC, the extent of the disease was assessed as proctitis (E1), left sided (E2), or extensive (E3) according to the Montreal classification as well.

\section{Statistical Analysis}

For categorical variables, Fisher exact or chi-square tests were used to assess the differences between the groups. For non-categorical variables, the Mann-Whitney $U$ test was used and they were described as medians and ranges. The treatments patterns were analyzed after controlling for different baseline clinical characteristics, such as initial symptoms, disease behavior, CD location, extent of UC, and age at diagnosis using propensity score matching between the 2 groups. Propensity scores were estimated using a logistic regression analysis. A Kaplan-Meier analysis with the log-rank test was applied to describe cumulative use of thiopurine and anti-TNF between the groups. A two-tailed $P$-value $<0.05$ was considered statistically significant. Statistical analyses were performed using SPSS version 18.0 (SPSS Inc., Chicago, IL, USA).

\section{RESULTS}

\section{Baseline Characteristics}

Overall, 178 patients with CD and 269 patients with UC were included in the study. For CD, 76 patients were from the SH while 102 were from the TRCs. The age at diagnosis for the CD patients was not different between the SH and the TRCs (median, 21 years (18-71) vs. 22 years (18-69); $P=0.371$ ). There was no difference in age of enrollment and follow-up period between SH and TRC. Both groups had a male predominance (SH $65.8 \%$ and TRC $76.5 \%, P=0.117$ ). There was a clear difference between the groups regarding their initial CD symptoms. Specifically, perianal symptoms were more frequently observed in the SH (56.6\% vs. $34.3 \%$, $P=0.003$ ) while luminal symptoms, such as abdominal pain $(17.1 \%$ vs. $54.9 \%, P<0.001)$, diarrhea $(18.4 \%$ vs. $44.1 \%$, $P<0.001)$, and body weight loss ( $1.3 \%$ vs. $9.8 \%, P=0.025)$ were more likely reported in the TRCs. Regarding the CD locations, ileal involvement (L1) was observed more in the TRC group than in the SH group (35.3\% vs. $19.7 \%, P=0.015$ ). Complicating behaviors, such as stricturing or penetrating types were more common in the TRCs than in the SH (35.3\% vs. $3.9 \%, P<0.001)$. The $\mathrm{SH}$ group had more perianal disease instances than the TRC group ( $80.3 \%$ vs. $57.8 \%, P=0.002$ ). 
Table 1. Baseline Characteristics of Patients with CD before and after Matching

\begin{tabular}{|c|c|c|c|c|c|c|}
\hline \multirow{2}{*}{ Characteristic } & \multicolumn{3}{|c|}{ Before matching } & \multicolumn{3}{|c|}{ After matching } \\
\hline & $\mathrm{SH}(n=76)$ & $\operatorname{TRC}(n=102)$ & $P$-value & $\mathrm{SH}(n=49)$ & $\operatorname{TRC}(n=49)$ & $P$-value \\
\hline Age of diagnosis (yr) & 21 & 22 & 0.371 & 20 & 20 & 0.760 \\
\hline Age of enrollment (yr) & 23 & 26 & 0.295 & 23 & 23 & 0.969 \\
\hline Follow-up (mo) & 19 & 21 & 0.133 & 20 & 24 & 0.258 \\
\hline Male sex & $50(65.8)$ & 78 (76.5) & 0.117 & $34(69.4)$ & 37 (75.5) & 0.498 \\
\hline \multicolumn{7}{|l|}{ Initial symptoms } \\
\hline Perianal symptoms & $43(56.6)$ & $35(34.3)$ & 0.003 & $25(51.0)$ & $23(46.9)$ & 0.840 \\
\hline Abdominal pain & $13(17.1)$ & $56(54.9)$ & $<0.001$ & $12(24.5)$ & $14(28.6)$ & 0.819 \\
\hline Diarrhea & $14(18.4)$ & $45(44.1)$ & $<0.001$ & $13(26.5)$ & $14(28.6)$ & 0.821 \\
\hline Body weight loss & $1(1.3)$ & $10(9.8)$ & 0.025 & $1(2.0)$ & $2(4.1)$ & 1.000 \\
\hline Location & & & 0.015 & & & 0.864 \\
\hline Ileal & 15 (19.7) & $36(35.3)$ & & $12(24.5)$ & $10(20.4)$ & \\
\hline Colonic & $21(27.6)$ & $33(32.4)$ & & $17(34.7)$ & $19(38.8)$ & \\
\hline Ileocolonic & $40(52.6)$ & $33(32.4)$ & & $20(40.8)$ & $20(40.8)$ & \\
\hline Behavior & & & $<0.001$ & & & 0.337 \\
\hline Inflammatory & 73 (96.1) & $66(64.7)$ & & 46 (93.9) & 44 (89.8) & \\
\hline Stricturing & $1(1.3)$ & $16(15.7)$ & & $1(2.0)$ & $4(8.2)$ & \\
\hline Penetrating & $2(2.6)$ & 20 (19.6) & & $2(4.1)$ & $1(2.0)$ & \\
\hline Perianal disease & 61 (80.3) & $59(57.8)$ & 0.002 & $36(73.5)$ & 33 (67.3) & 0.658 \\
\hline
\end{tabular}

Values are presented as median or number (\%).

$\mathrm{SH}$, secondary hospital; TRC, tertiary referral center.

Table 2. Baseline Characteristics of Patients with UC before and after Matching

\begin{tabular}{|c|c|c|c|c|c|c|}
\hline \multirow{2}{*}{ Characteristic } & \multicolumn{3}{|c|}{ Before matching } & \multicolumn{3}{|c|}{ After matching } \\
\hline & SH $(n=170)$ & TRC $(n=99)$ & $P$-value & SH $(n=65)$ & $\operatorname{TRC}(n=65)$ & $P$-value \\
\hline Age of diagnosis (yr) & 47 & 36 & $<0.001$ & 53 & 41 & 0.162 \\
\hline Age of enrollment (yr) & 52 & 39 & 0.001 & 57 & 49 & 0.196 \\
\hline Follow-up (mo) & 36 & 20 & 0.012 & 37 & 22 & 0.127 \\
\hline Male sex & $113(66.5)$ & $56(56.6)$ & 0.117 & $49(75.4)$ & $40(61.5)$ & 0.131 \\
\hline \multicolumn{7}{|l|}{ Initial symptoms } \\
\hline Hematochezia & $159(93.5)$ & 77 (77.8) & $<0.001$ & 58 (89.2) & $59(90.8)$ & 1.000 \\
\hline Diarrhea & $40(23.5)$ & $56(56.6)$ & $<0.001$ & $20(30.8)$ & 27 (41.5) & 0.273 \\
\hline Abdominal pain & $12(7.1)$ & $16(16.2)$ & 0.023 & 7 (10.8) & $5(7.7)$ & 0.545 \\
\hline Body weight loss & 0 & $8(8.1)$ & 0.001 & 0 & 0 & \\
\hline Disease extent & & & $<0.001$ & & & 0.120 \\
\hline Proctitis & $100(58.8)$ & $21(21.2)$ & & $17(26.2)$ & $17(26.2)$ & \\
\hline Left sided & 33 (19.4) & $50(50.5)$ & & $22(33.8)$ & $32(49.2)$ & \\
\hline Extensive & 37 (21.8) & $28(28.3)$ & & $26(40.0)$ & $16(24.6)$ & \\
\hline
\end{tabular}

Values are presented as median or number (\%).

$\mathrm{SH}$, secondary hospital; TRC, tertiary referral center. 
Regarding UC, 170 SH patients and 99 TRC patients were evaluated. The ages at diagnosis in the SH were significantly higher than in the TRCs (median, 47 years vs. 36 years; $P<0.001)$. There was no significant difference in gender between the groups. We found a significant difference in the age of enrollment (median, 52 years vs. 39 years; $P=0.001$ ) and follow-up duration (median, 36 months vs. 20 months; $P=0.012$ ) between SH and TRC before matching. Although hematochezia was the most commonly reported initial symptom in both groups, the SH patients had this symptom more frequently than the TRC patients did (93.5\% vs. $77.8 \%, P<0.001)$. However, the UC patients in the TRCs more frequently reported diarrhea ( $56.6 \%$ vs. $23.5 \%, P<0.001$ ), abdominal pain ( $16.2 \%$ vs. $7.1 \%, P=0.023$ ) and body weight loss ( $8.1 \%$ vs. $0 \%, P=0.001)$ than in the SH. The UC patients from the SH had a more limited extent of the disease than in the TRCs (proctitis $58.8 \%$ vs. $21.2 \%, P<0.001$ ). Statistically significant differences in the baseline characteristics including initial symptoms, CD location, CD behavior, age at diagnosis and enrollment of UC, follow-up duration in UC and extent of UC were not observed after propensity score matching.

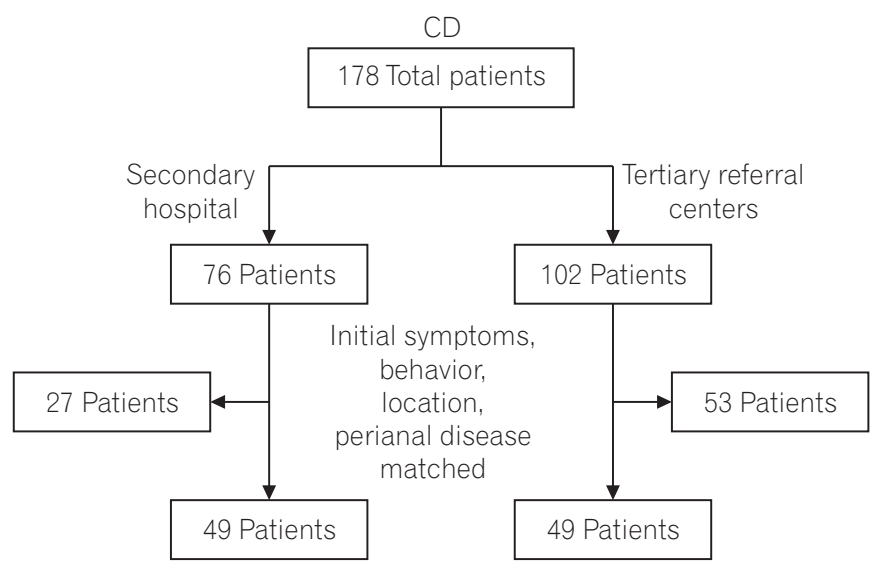

The baseline characteristics are described in Tables 1 and 2 .

\section{Treatments Patterns between the Groups after Matching}

Before comparing the treatments pattern between the groups, we matched patients at a 1 to 1 ratio from each group using propensity score matching (Fig. 1). The number of patients was reduced to 98 ( 49 vs. 49) and 130 (65 vs. 65) for $\mathrm{CD}$ and UC, respectively. The treatment patterns between the groups were compared, as shown in Table 3. The CD patients in the TRCs were exposed to steroids more frequently than in the $\mathrm{SH}$ ( $42.9 \%$ vs. $12.2 \%, P=0.001$ ). Additionally, more CD patients in the TRCs took thiopurine medication than in the SH $(87.8 \%$ vs. $16.3 \%, P<0.001)$. However, the use of antiTNF for CD in the SH was similar to that in the TRCs (30.6\% vs. $24.5 \%, P=0.498$ ). Anti-TNF treatment without thiopurine initiation was more common in the SH than in the TRCs ( $20.4 \%$ vs. $0 \%, P=0.001)$. Bowel resection rate was not different between groups. For the UC treatment, there was no significant difference between the groups regarding steroid

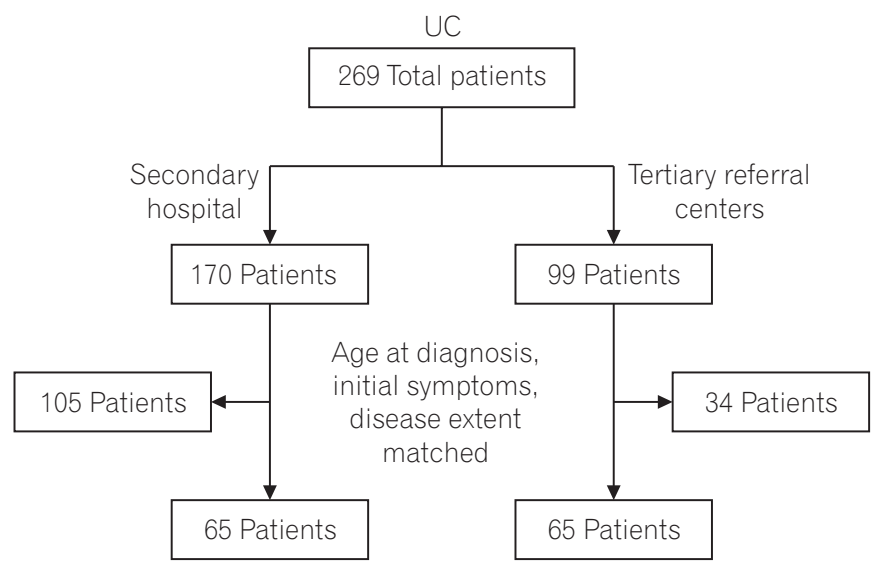

Fig. 1. Matching flow of the patients.

Table 3. Treatment Pattern in a Secondary Hospital and Tertiary Referral Centers after Matching

\begin{tabular}{|c|c|c|c|c|c|c|}
\hline \multirow{2}{*}{ Treatments } & \multicolumn{3}{|c|}{$C D(n=98)$} & \multicolumn{3}{|c|}{ UC $(n=130)$} \\
\hline & SH $(n=49)$ & $\operatorname{TRC}(n=49)$ & $P$-value & SH $(n=65)$ & $\operatorname{TRC}(n=65)$ & $P$-value \\
\hline Steroid exposure & $6(12.2)$ & $21(42.9)$ & 0.001 & $24(36.9)$ & $28(43.1)$ & 0.474 \\
\hline Thiopurine & $8(16.3)$ & 43 (87.8) & $<0.001$ & $8(12.3)$ & $20(30.8)$ & 0.018 \\
\hline Anti-TNF & $15(30.6)$ & $12(24.5)$ & 0.498 & $9(13.8)$ & $7(10.8)$ & 0.790 \\
\hline Anti-TNF without thiopurine use & $10(20.4)$ & 0 & 0.001 & $4(6.2)$ & $1(1.5)$ & 0.365 \\
\hline Surgery $^{a}$ & $4(8.2)$ & $1(2.0)$ & 0.362 & 0 & $1(1.5)$ & 1.000 \\
\hline
\end{tabular}

Values are presented as number (\%).

aSurgery indicates bowel resection and colectomy for $C D$ and $U C$, respectively.

SH, secondary hospital; TRC, tertiary referral center; anti-TNF, anti-tumor necrosis factor. 

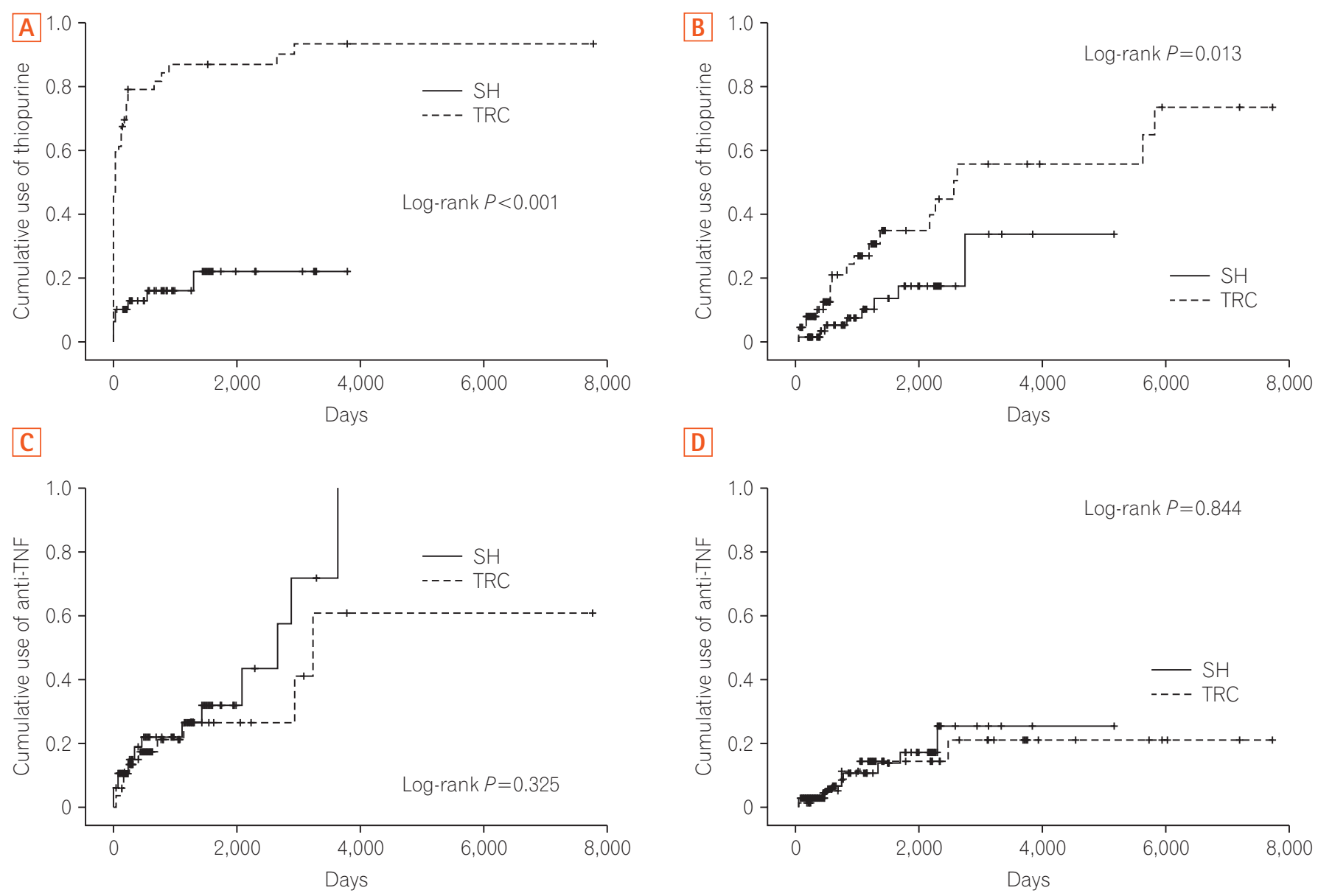

Fig. 2. Kaplan-Meier analysis of cumulative use of treatment after matching. (A) Thiopurine in $C D,(B)$ thiopurine in UC, $(C)$ anti-tumor necrosis factor (anti-TNF) in CD, and (D) anti-TNF in UC. SH, secondary hospital; TRC, tertiary referral centers.

exposure and anti-TNF use, whereas more patients in the TRCs were administered thiopurine medication than in the SH (30.8\% vs. $12.3 \%, P=0.018)$. Total colectomy rate was not different between groups. A Kaplan-Meier analysis of the cumulative use of thiopurine showed that the time from the diagnosis to start of thiopurine treatment was significantly shorter in the TRC group compared with the SH group for both CD ( $\log$-rank $P<0.001)$ and UC $(\log -\operatorname{rank} P=0.013)$ (Fig. $2 \mathrm{~A}$ and $\mathrm{B})$. We did not find a significant difference between the groups in the cumulative use of anti-TNF for both CD and UC (Fig. 2C and D).

\section{DISCUSSION}

We found that there was a significant difference in the clinical characteristics of patients with IBD from a secondary care unit and from TRCs. The CD patients in the SH were more likely to have perianal disease-related problems whereas the CD patients in the TRCs had ileal location and complicating behaviors more frequently compared with the SH. Additionally, the UC patients in the SH were more likely to have a limited extent of the disease compared with those in the TRCs. Moreover, there was a substantial gap in the treatment behavior for patients with IBD between the SH and TRCs. After controlling for different characteristics using propensity score matching, the cumulative use of thiopurine for both CD and UC in the SH group was significantly lower than that in the TRC group.

To the best of our knowledge, this is the first study to compare the clinical features and management of patients with IBD between a secondary care unit and TRCs using a propensity score matching system. Although IBD is perceived as a condition that usually requires management by gastroenterology specialists in referral centers, the role of primary or secondary care units is recognized as essential in the longterm care of patients with IBD. ${ }^{8}$ Further, issues in the communication between health care services for patients with IBD have been highlighted. ${ }^{9}$ Therefore, it is imperative to 
understand the characteristics and management behaviors of patients with IBD before they get to referral centers.

Interestingly, this study showed that the SH group had more CD patients who had perianal fistulas than in the TRCs. Although the exact cause is unclear, accessibility to each health care service might contribute to this difference. For instance, patients who had anal discharge or painful anal swelling usually go to the Department of Surgery and, from the patients' perspective, secondary care hospitals are more accessible than TRCs. This finding is noteworthy because perianal disease is known as a peculiar feature of Korean CD patients as they more frequently have perianal disease than in Western countries. ${ }^{10}$ In contrast, complicating behaviors, such as stricturing or penetrating, and ileal location were more frequently observed in the TRC group. This is not surprising, as referral centers address more serious patients who are referred from primary or SHs. Ileal location is a wellknown indicator of poor clinical CD outcomes. ${ }^{11}$ The result that the SH was more likely to have a limited extent of UC is also a reflection of this aspect, as proctitis is related to a mild UC disease course. ${ }^{12}$ Likewise, the UC patients in the TRCs were younger at the time of diagnosis than those of the $\mathrm{SH}$, indicating more adverse clinical outcomes in the TRC group, as younger diagnosis ages are known to be related with poor clinical outcomes for UC..$^{13,14}$

Regarding the UC patients, steroid exposure was not different between the SH and TRC groups after controlling for baseline characteristics with propensity score matching. However, cumulative thiopurine use for UC in the SH was significantly lower than in the TRCs (Fig. 2B). This was also true for the management of $\mathrm{CD}$. Cumulative thiopurine use for $\mathrm{CD}$ in the SH was much lower than the TRCs after matching between the groups (Fig. 2A). In addition, the use of antiTNF without thiopurine therapy was more common in the SH than in the TRCs ( $20.4 \%$ vs. $0 \%$ for CD; $6.2 \%$ vs. $1.5 \%$ for UC) (Table 3). Although the exact reason of the underuse of thiopurine in the $\mathrm{SH}$ is unknown, it might be explained by the considerable adverse effects of thiopurine, including bone marrow suppression, hepatotoxicity, and increased risk of opportunistic infections and malignancies, which are difficult for physicians in SHs to handle. ${ }^{8}$ In particular, leukopenia occurrences are the main obstacle to thiopurine use in clinical practice, as it requires tight laboratory white blood cell monitoring. ${ }^{15}$ Indeed, the risk of thiopurine-induced leukopenia is much higher in Asians, including Koreans $(\sim 35.4 \%),)^{16,17}$ than in Western countries $\left.(\sim 5 \%)\right)^{18,19}$ Therefore, Korean physicians might be more reluctant to use thiopurine for IBD management in their clinical practice. Another plausible explanation for inadequate use of thiopurine in the $\mathrm{SH}$ might be related with the Korean medical reimbursement system. According to the Korean National Health Insurance Service, thiopurine failure is required before anti-TNF therapy for luminal CD, indicating that thiopurine treatment is prerequisite for use of anti-TNF. However, thiopurine treatment is not necessarily mandatory before anti-TNF therapy for perianal CD or UC. Hence, the use of anti-TNF seems to be more liable without consideration of response to thiopurine in perianal CD or UC than luminal CD in Korea.

A robust effort is required to determine the exact hurdles for the underuse of thiopurine for IBD management by physicians in primary or secondary care units and to provide them with education for optimal thiopurine use. Recently, a consensus statement has been developed for guiding the appropriate use of thiopurine in IBD, which are more suitable for Korean patients. ${ }^{20}$ Further, several genetic biomarkers for predicting thiopurine-induced leukopenia confirmed in Korean studies might be helpful to use thiopurine properly in the management of Korean patients with IBD. ${ }^{16,21}$ These genetic biomarkers are particularly useful for physicians working at $\mathrm{SH}$ in the light of their concern of thiopurine-induced leukopenia.

This study had several limitations. First, there might be a selection bias inherent to the retrospective study design. Second, although we used a propensity score matching method to control for the disease phenotypes, it might not have fully regulated the differences in disease severity. However, a validated assessment of disease severity was not available in the retrospective setting. A successful control of disease severity between the groups in our study might be reflected by a similar anti-TNF use rate and surgery rate in both groups after propensity matching, which are one of indicators for disease severity. ${ }^{22}$ Third, the SH in this study is specialized in coloproctology although it has many other departments as well. Therefore, it might be difficult to generalize data of the study. Further study with more data from primary or secondary care hospitals is needed.

In conclusion, the clinical characteristics and management of patients with IBD in the SH were different from the TRCs. The SH was more focused on the perianal disease for $\mathrm{CD}$, whereas the TRCs had more complicating behaviors and ileal location. For UC, patients in the SH had a more limited extent compared with those in the TRCs. Overall, patients with IBD in the SH were less likely to receive treatment with thiopurine than those in the TRCs after controlling for different baseline characteristics using propensity score matching. Therefore, this study indicates that there is room for improvement in the proper use of thiopurine therapy in the SH. 


\section{FINANCIAL SUPPORT}

This work was supported by Basic Science Research Program through the National Research Foundation of Korea (NRF) funded by the Ministry of Education (2015R1D1A1A02062168).

\section{CONFLICT OF INTEREST}

No potential conflict of interest relevant to this article was reported.

\section{AUTHOR CONTRIBUTION}

Research concepts and design: Kim ES. Data acquisition: Kim KO, Jang BI, Song KH, Data analysis and interpretation: Kim ES, Jang BI, Lee HS. Statistical analysis: Kwak SG, Lee YJ. Drafting the manuscript: Song KH, Kim ES. Critical revision of the manuscript: Jang BI. Receiving grant: Kim ES. Approval of final manuscript: all authors.

\section{REFERENCES}

1. Baumgart DC, Bernstein CN, Abbas Z, et al. IBD Around the world: comparing the epidemiology, diagnosis, and treatment: proceedings of the World Digestive Health Day 2010: Inflammatory Bowel Disease Task Force meeting. Inflamm Bowel Dis 2011;17:639-644.

2. Thia KT, Loftus EV Jr, Sandborn WJ, Yang SK. An update on the epidemiology of inflammatory bowel disease in Asia. Am J Gastroenterol 2008;103:3167-3182.

3. Ng SC, Tang W, Ching JY, et al. Incidence and phenotype of inflammatory bowel disease based on results from the AsiaPacific Crohn's and Colitis Epidemiology Study. Gastroenterology 2013;145:158-165.e2.

4. Ng WK, Wong SH, Ng SC. Changing epidemiological trends of inflammatory bowel disease in Asia. Intest Res 2016;14:111119.

5. Tursi A, Elisei W, Picchio M. Incidence and prevalence of inflammatory bowel diseases in gastroenterology primary care setting. Eur J Intern Med 2013;24:852-856.

6. Ye BD, Jang BI, Jeen YT, et al. Diagnostic guideline of Crohn's disease. Korean J Gastroenterol 2009;53:161-176.

7. Choi CH, Jung SA, Lee BI, et al. Diagnostic guideline of ulcerative colitis. Korean J Gastroenterol 2009;53:145-160.

8. Thomas A, Lodhia N. Advanced therapy for inflammatory bowel disease: a guide for the primary care physician. J Am Board Fam Med 2014;27:411-420.
9. Torjesen I. Care of IBD patients compromised by poor communication between primary and secondary care. BMJ 2012;344:e2675. doi: 10.1136/bmj.e2675.

10. Ye BD, Yang SK, Cho YK, et al. Clinical features and long-term prognosis of Crohn's disease in Korea. Scand J Gastroenterol 2010;45:1178-1185.

11. Bernell O, Lapidus A, Hellers G. Risk factors for surgery and postoperative recurrence in Crohn's disease. Ann Surg 2000;231:38-45

12. Reenaers C, Pirard C, Vankemseke C, Latour P, Belaiche J, Louis E. Long-term evolution and predictive factors of mild inflammatory bowel disease. Scand J Gastroenterol 2016;51:712-719.

13. Riegler G, Tartaglione MT, Carratú R, et al. Age-related clinical severity at diagnosis in 1705 patients with ulcerative colitis: a study by GISC (Italian Colon-Rectum Study Group). Dig Dis Sci 2000;45:462-465.

14. Choi JH, Kim ES, Cho KB, et al. Old age at diagnosis is associated with favorable outcomes in Korean patients with inflammatory bowel disease. Intest Res 2015;13:60-67.

15. Fischer SH, Tjia J, Reed G, Peterson D, Gurwitz JH, Field TS. Factors associated with ordering laboratory monitoring of high-risk medications. J Gen Intern Med 2014;29:1589-1598.

16. Yang SK, Hong M, Baek J, et al. A common missense variant in NUDT15 confers susceptibility to thiopurine-induced leukopenia. Nat Genet 2014;46:1017-1020.

17. Takatsu N, Matsui T, Murakami Y, et al. Adverse reactions to azathioprine cannot be predicted by thiopurine S-methyltransferase genotype in Japanese patients with inflammatory bowel disease. J Gastroenterol Hepatol 2009;24:1258-1264.

18. Connell WR, Kamm MA, Ritchie JK, Lennard-Jones JE. Bone marrow toxicity caused by azathioprine in inflammatory bowel disease: 27 years of experience. Gut 1993;34:1081-1085.

19. Fraser AG, Orchard TR, Jewell DP. The efficacy of azathioprine for the treatment of inflammatory bowel disease: a 30 year review. Gut 2002;50:485-489.

20. Lee KM, Kim YS, Seo GS, Kim TO, Yang SK; IBD Study Group of the Korean Association for the Study of Intestinal Diseases. Use of thiopurines in inflammatory bowel disease: a consensus statement by the Korean Association for the Study of Intestinal Diseases (KASID). Intest Res 2015;13:193-207.

21. Kim HS, Cheon JH, Jung ES, et al. A coding variant in FTO confers susceptibility to thiopurine-induced leukopenia in East Asian patients with IBD. Gut 2017;66:1926-1935.

22. Sulz MC, Siebert U, Arvandi M, et al. Predictors for hospitalization and outpatient visits in patients with inflammatory bowel disease: results from the Swiss Inflammatory Bowel Disease Cohort Study. Eur J Gastroenterol Hepatol 2013;25:790-797. 